\title{
On the mean free path for a periodic array of spherical obstacles
}

\author{
H.S. DUMAS ${ }^{1}$, L. DUMAS ${ }^{2}$, and F. GOLSE ${ }^{2}$ \\ ${ }^{1}$ Dept. of Mathematical Sciences \\ University of Cincinnati \\ Cincinnati, $\mathrm{OH} 45221-0025$ \\ USA \\ ${ }^{2}$ UFR de Mathématiques \\ Université Paris VII \\ Tour $45-55,5^{\mathrm{e}}$ étage \\ 4 place Jussieu \\ 75251 Paris Cedex 05 \\ France
}

\begin{abstract}
We prove theorems pertaining to periodic arrays of spherical obstacles which show how the macroscopic limit of the mean free path depends on the scaling of the size of the obstacles. We treat separately the cases where the obstacles are totally and partially absorbing, and we also distinguish between two-dimensional arrays, where our results are optimal, and higher dimensional arrays where they are not. The cubically symmetric arrays to which these results apply do not have finite horizon.
\end{abstract}

\section{Introduction.}

The idea of the "mean free path" for large ensembles of particles interacting among themselves (or moving among obstacles or within an enclosure) is quite intuitive, and lies at the foundations of kinetic theory as conceived by Maxwell and Boltzmann in the late nineteenth century. For a given physical system, estimates of the mean free path are important in determining the sort of dynamics that predominates. However, actually calculating the mean free path-even in many simplified models-is problematic, and it is often necessary to resort to dimensional arguments that are not wholly rigorous. One family of simplified models that has played an important role in statistical mechanics and kinetic theory is the so-called Lorentz gas in a plane dispersive billiard consisting of a periodic array of circular obstacles, together with its higher dimensional analogues. In kinetic theory, it is particularly interesting to study the behavior of particles in such billiards in the macroscopic limit (i.e., as the size of the array is reduced to zero). In this article, we prove theorems pertaining to periodic arrays of spherical obstacles which show how the macroscopic limit of the mean free path depends on the scaling of the size of the obstacles. We treat separately the cases where the obstacles are totally and partially absorbing, and we also distinguish between two-dimensional arrays, where our results are optimal, and higher dimensional arrays where they are not. The interesting aspect of these results is that the (hyper-) cubically symmetric arrays to which they apply do not have finite horizon (i.e., the arrays have unbounded trajectories).

The Lorentz gas has been widely studied: in Refs. [4] and [5], Bunimovich and Sinai, then Bunimovich, Sinai, and Chernov construct (analogues of) Markov partitions to analyze the "hydrodynamic limit" (in this case a Brownian motion) of a Lorentz gas with finite horizon. But the hydrodynamic limit corresponds to cases where the mean free path 
tends to zero, something that is assured in [4] and [5] by the scaling and the geometric hypothesis of finite horizon. The "kinetic" regime (also known as the Boltzmann-Grad limit) corresponds to cases where the limiting value of the mean free path is of the order of the unit length: hence this limit appears for periodic Lorentz gases only in cases where the finite horizon hypothesis does not hold. The Boltzmann-Grad limit of the Lorentz gas has been studied by many authors, but essentially only for random distributions of obstacles; see Galavotti [9], Spohn [16], and Boldighrini, Bunimovich, and Sinai [3]. The Boltzmann-Grad limit for clouds of interacting particles (leading to the nonlinear Boltzmann equation) was investigated on a rigorous basis by Lanford [12], and subsequently by Illner and Pulvirenti [11] (in the two dimensional case), and by Pulvirenti [13].

However, as can be seen from the discussion below, the case of a square (or cubic, or hypercubic) array of spherical obstacles involves number theoretic questions (essentially rational approximation) in a structural way. Because of this, the results we find in (space) dimensions higher than two are not as good as those we find in two dimensions. The higher dimensional case involves "simultaneous rational approximation" (of several real numbers by fractions with the same denominator), while the two-dimensional case entails approximation of one real number by a sequence of rationals, where the algorithm of continued fractions is known to be in some sense optimal. For discussions of these questions, we refer the interested reader to Cassels [6] and Schmidt [14].

\section{The $n$-Dimensional Dispersive Billiard and its Mean Free Path}

The $n$-dimensional analogue of a periodic, planar array of circular scatterers with square symmetry is simply the periodic array of spherical obstacles defined as follows. First, denote by $\mathcal{L}_{\varepsilon} \equiv a \varepsilon \mathbf{Z}^{n}$ the (hyper-) cubic lattice in $\mathbf{R}^{n}$ with interstitial spacing $a \varepsilon$. The billiard domain is then

$$
Z_{\varepsilon}=\left\{x \in \mathbf{R}^{n} \mid \operatorname{dist}\left\{x, \mathcal{L}_{\varepsilon}\right\}>r \varepsilon^{\gamma}\right\}
$$

Here $\varepsilon>0$ is a small parameter which controls the spacing between obstacles in the array, while the exponent $\gamma$ controls the way the size of the obstacles scales with $\varepsilon$ (we assume that $\gamma \geq 1$ and $0<2 r<a$ so that obstacles do not overlap in the macroscopic limit $\varepsilon \rightarrow 0)$.

Using dimensional arguments, it is possible to estimate the order of magnitude of the mean free path for a population of point particles moving between the obstacles with constant speed $c$ (neglecting collisions between particles). To see this, note that for a given geometry, the mean free path diminishes as the volume-density of obstacles-or as the size of the obstacles-increases. It therefore seems plausible to suppose that the order of magnitude of the mean free path is given by

$$
\frac{1}{N_{\varepsilon} S_{\varepsilon}}
$$

where $N_{\varepsilon}$ is the density of obstacles per unit $n$-dimensional volume and $S_{\varepsilon}$ is an $n-1$ dimensional volume element (so that the above expression has the dimension of length) 
measuring the size of the obstacles. For example, for $S_{\varepsilon}$, one could take the $n-1$ dimensional volume (physically, the "surface area") of the obstacles, so that $S_{\varepsilon}=$ $\left|\mathbf{S}^{n-1}\right| r^{n-1} \varepsilon^{\gamma(n-1)}$ (here $\left|\mathbf{S}^{n-1}\right|$ denotes the measure of $\mathbf{S}^{n-1}$; the only measure we consider on $\mathbf{S}^{n-1}$ is the Riemannian density associated to the metric induced on $\mathbf{S}^{n-1}$ by the Euclidean metric on $\mathbf{R}^{n}$, so that $\left|\mathbf{S}^{1}\right|=2 \pi,\left|\mathbf{S}^{2}\right|=4 \pi^{2}$, etc.). Using this ansatz together with (2), we arrive to the following estimate of the order of magnitude of the mean free path in $Z_{\varepsilon}$ :

$$
\frac{a^{n}}{\left|\mathbf{S}^{n-1}\right| r^{n-1}} \varepsilon^{n-\gamma(n-1)} \text {. }
$$

The same expression is found after a more detailed analysis using diffusive billiard dynamics for $Z_{\varepsilon}$ in the sense of "weak consistency" (see Golse [10]). Regardless of the process used to obtain the estimate of the mean free path (3), it suggests that the value $\gamma_{c}=\frac{n}{n-1}$ of the parameter $\gamma$ is critical in the following sense:

- For $1 \leq \gamma<\gamma_{c}$, the mean free path tends to zero with $\varepsilon$. Assuming specular reflection of particles from the obstacles, one expects the movement of the particles in $Z_{\varepsilon}$ to be given by an equation of hydrodynamic type (see Bunimovich-Sinai [4]).

- For $\gamma>\gamma_{c}$, the mean free path tends to infinity as $\varepsilon \rightarrow 0$. It is then trivial to show that the motion of the particles in $Z_{\varepsilon}$ is governed by a free-transport equation.

- For $\gamma=\gamma_{c}$, the mean free path is of order 1 as $\varepsilon \rightarrow 0$, and one expects the motion of the particles in $Z_{\varepsilon}$ to be described by a kinetic equation.

Nevertheless, no rigorous calculation of the mean free path in $Z_{\varepsilon}$ exists, in part because of the presence of unbounded trajectories (e.g. some trajectories parallel to the axes of the lattice $\mathcal{L}_{\varepsilon}$ are unbounded), and the presence of arbitrarily long trajectories. In the sequel, we shall substantiate the intuition of formula (3) with explicit calculations.

\section{Transport Equation Formalism.}

We shall consider two types of problems: Case A, where particles are totally absorbed at the boundary of $Z_{\varepsilon}$; and Case $\mathrm{B}$, where, upon reaching the boundary of $Z_{\varepsilon}$, particles are partially absorbed, then reflected with coefficient of reflection $\alpha(0<\alpha<1$; our methods unfortunately do not apply to the case of total reflection $\alpha=1$ ).

Let $f_{\varepsilon}(t, x, \omega)$ be the density of particles at the point $x$, at time $t$, moving in the direction $\omega \in \mathbf{S}^{n-1}$. We write

$$
\Gamma_{\varepsilon}^{+}=\left\{(x, \omega) \in \partial Z_{\varepsilon} \times \mathbf{S}^{n-1} \mid \omega \cdot n_{x}>0\right\},
$$

where $n_{x}$ is the inward normal at the point $x \in \partial Z_{\varepsilon}$. (Here "inward" means toward the interior of $Z_{\varepsilon}$; away from the centers of the balls $Z_{\varepsilon}^{c}$.) The equation satisfied by $f_{\varepsilon}$ is

$$
\partial_{t} f_{\varepsilon}+c \omega \cdot \nabla_{x} f_{\varepsilon}=0, \quad x \in Z_{\varepsilon}
$$

with initial data

$$
f_{\varepsilon}(0, x, \omega)=\phi(x, \omega), \quad x \in Z_{\varepsilon}
$$

and with either the condition of totally absorbing boundaries (Case A):

$$
f_{\varepsilon}(t, x, \omega)=0, \quad(x, \omega) \in \Gamma_{\varepsilon}^{+}
$$


or the condition of partially absorbing/partially reflecting boundaries (Case B):

$$
f_{\varepsilon}(t, x, \omega)=\alpha f_{\varepsilon}\left(t, x, \mathcal{R}\left(n_{x}\right) \omega\right), \quad(x, \omega) \in \Gamma_{\varepsilon}^{+}
$$

Here $\mathcal{R}\left(n_{x}\right) \omega$ represents specular reflection: $\mathcal{R}\left(n_{x}\right) \omega=\omega-2\left(\omega \cdot n_{x}\right) n_{x}$; and $\phi$ is a nonnegative function defined on the whole of $\mathbf{R}^{n} \times \mathbf{S}^{n-1}$.

We may write the solution of (5)-(6)-(7A) using the method of characteristics. Let $\tau_{\varepsilon}(x, \omega)$ be the time of exit from $Z_{\varepsilon}$, in other words

$$
\tau_{\varepsilon}(x, \omega)=\inf \left\{t>0 \mid x-t c \omega \in \partial Z_{\varepsilon}\right\}
$$

For fixed $x \in Z_{\varepsilon}, \tau_{\varepsilon}(x, \omega)$ is finite for almost every $\omega \in \mathbf{S}^{n-1}$. (In fact, for "irrational" $\omega$; that is, $\omega$ such that $\forall k \in \mathbf{Z}^{n} \backslash\{0\}, k \cdot \omega \neq 0$ it is well known that the trajectory of every point of the torus $\mathbf{T}^{n} \equiv \mathbf{R}^{n} / \mathbf{Z}^{n}$ is dense in $\mathbf{T}^{n}$; in other words, the linear flow in the direction $\omega$ on $\mathbf{T}^{n}$ is topologically transitive.) The solution of (4)-(5)-(6A) is given by

$$
f_{\varepsilon}(t, x, \omega)=\mathbf{1}_{0 \leq t<\tau_{\varepsilon}(x, \omega)} \phi(x-t c \omega, \omega)
$$

and this formula shows that if $\tau_{\varepsilon}(x, \omega) \rightarrow 0$ as $\varepsilon \rightarrow 0$, then $f_{\varepsilon}(t, x, \omega) \rightarrow 0$. On the other hand, if, for any initial data $\phi$ bounded on $\mathbf{R}^{n}$ we had $f_{\varepsilon} \rightarrow 0$ as $\varepsilon \rightarrow 0$, this would mean that the equivalent effective cross section of absorption (as $\varepsilon \rightarrow 0$ ) of the array of obstacles $Z_{\varepsilon}$ is infinite, or, what amounts to the same thing, that the mean free path tends to 0 with $\varepsilon$. Formula (8) therefore shows that in order to establish that $\gamma<\gamma_{c}$, it suffices to show that $f_{\varepsilon} \rightarrow 0$ for the initial data $\phi=1$.

\section{Principal Results.}

We give several results affirming formula (3). For two-dimensional arrays, we state theorems implying $\gamma_{c}=2$. For higher dimensional arrays, we state similar but slightly weaker theorems which show that $\frac{n}{n-2 / 3} \leq \gamma_{c} \leq \frac{n}{n-1}$. As before, we divide these statements into those for totally absorbing obstacles, and those for partially absorbing obstacles.

\section{A.The Case of Total Absorption}

As mentioned above, the best result concerns the case where the dimension of the space is two.

Theorem 1 A. Let $n=2$, and choose $1 \leq \gamma<2, T>0$, and a compact "observation set" $K \subset \mathbf{R}^{2}$. Then given any number $s$ with $1<s<(\gamma-1)^{-1}$, there exists a constant $A$ such that for any initial data $\phi \in L^{\infty}\left(\mathbf{R}^{2} \times \mathbf{S}^{1}\right)$, the family $f_{\varepsilon}$ of solutions of the problem (5)-(6)-(7A) satisfies

$$
\left|\int_{0}^{T} \int_{K \cap Z_{\varepsilon}} \int_{\mathbf{S}^{1}}\right| f_{\varepsilon}(t, x, \omega)|d \omega d x d t| \leq A \varepsilon^{(1+s-\gamma s) / 2}\|\phi\|_{L^{\infty}} .
$$

The proof of this theorem (as well as the proofs of those below) relies on certain estimates of the "ergodization time for linear flows on tori" which we discuss (and prove 
in the two-dimensional case) in Section 5. These two-dimensional estimates are optimal in a sense which is clarified in Section 5. However, for tori of dimension greater than two, the estimates available on ergodization times are less precise. This is because in two dimensions, they are obtained using a continued fraction expansion of the slope of the direction vector, which is known to be an optimal approximation. In higher dimensions, this method no longer works, as it leads to a problem of simultaneous approximation of several irrationals by rational numbers.

Despite the lack of sharpness of the higher-dimensional estimates due to H.S. Dumas [8], our result based on them is as follows:

Theorem 2 A. Let $n>2$, and choose $1 \leq \gamma<\frac{n}{n-2 / 3}, T>0$, and a compact set $K \subset \mathbf{R}^{n}$. Then given any number $s$ with $1<s<(\gamma-1)^{-1}$, there exists a constant $A$ such that for any initial data $\phi \in L^{\infty}\left(\mathbf{R}^{n} \times \mathbf{S}^{n-1}\right)$, the family $f_{\varepsilon}$ of solutions of the problem (5)-(6)-(7A) satisfies

$$
\left|\int_{0}^{T} \int_{K \cap Z_{\varepsilon}} \int_{\mathbf{S}^{n-1}}\right| f_{\varepsilon}(t, x, \omega)|d \omega d x d t| \leq A \varepsilon^{(1+s-\gamma s) / 2}\|\phi\|_{L^{\infty}} .
$$

\section{B. The Case of Partial Absorption/Reflection}

We next state theorems for partially absorbing obstacles which closely parallel those for totally absorbing obstacles. However, the proofs of the theorems below are considerably more complicated, and lead to less precise estimates of the rate of decay of the mean free path.

Theorem 1B. Let $n=2$, and choose $1 \leq \gamma<2,0 \leq \alpha<1, T>0$, and a compact set $K \subset \mathbf{R}^{2}$. Then there exist constants $b>0, B>0$, and $\varepsilon_{0}>0$ such that for any initial data $\phi \in L^{\infty}\left(\mathbf{R}^{2} \times \mathbf{S}^{1}\right)$, and for $0<\varepsilon \leq \varepsilon_{0}$, the family $f_{\varepsilon}$ of solutions of the problem (5)-(6)-(7B) satisfies

$$
\left|\int_{0}^{T} \int_{K \cap Z_{\varepsilon}} \int_{\mathbf{S}^{1}}\right| f_{\varepsilon}(t, x, \omega)|d \omega d x d t| \leq B \varepsilon^{b}\|\phi\|_{L^{\infty}} .
$$

Theorem 2B. Let $n>2$, and choose $1 \leq \gamma<\frac{n}{n-2 / 3}, 0 \leq \alpha<1, T>0$, and a compact set $K \subset \mathbf{R}^{n}$. Then there exist constants $b>0, B>0$, and $\varepsilon_{0}>0$ such that for any initial data $\phi \in L^{\infty}\left(\mathbf{R}^{n} \times \mathbf{S}^{n-1}\right)$, and for $0<\varepsilon \leq \varepsilon_{0}$, the family $f_{\varepsilon}$ of solutions of the problem (5)-(6)-(7B) satisfies

$$
\left|\int_{0}^{T} \int_{K \cap Z_{\varepsilon}} \int_{\mathbf{S}^{n-1}}\right| f_{\varepsilon}(t, x, \omega)|d \omega d x d t| \leq B \varepsilon^{b}\|\phi\|_{L^{\infty}} .
$$

Remark 1. These results partly correct the errors that appear in the Note [1].

Remark 2. Theorems $1 \mathrm{~A}$ and $2 \mathrm{~A}$ (total absorption) apply to a more general class of scatterers than the spheres described in Eq. (1). In fact, Theorems $1 \mathrm{~A}$ and $2 \mathrm{~A}$ clearly apply to any class of scatterers which contains the scatterers defined as the complement of $Z_{\varepsilon}$ in Eq. (1). 
Remark 3. Using elementary distributional calculus, it is not difficult to show that for any dimension $n, \gamma_{c} \leq \frac{n}{n-1}$. It therefore follows from Theorems $1 \mathrm{~A}$ and $1 \mathrm{~B}$ that, in 2 dimensions, $\gamma_{c}=2$, as predicted by dimensional analysis (2), (3). On the other hand, Theorems $2 \mathrm{~A}$ and $2 \mathrm{~B}$ show that $\frac{n}{n-2 / 3} \leq \gamma_{c} \leq \frac{n}{n-1}$ in higher dimensions.

To see that $\gamma_{c} \leq \frac{n}{n-1}$, first note that if $\alpha=1$ (total reflection) is allowed in Eq. (7B), then by the Maximum Principle, nonnegative initial data $\phi \geq 0$ give rise to nonnegative solutions $f_{\varepsilon} \geq 0$ with $\left\|f_{\varepsilon}\right\|_{L^{\infty}} \leq\|\phi\|_{L^{\infty}}$ for all $t \geq 0$. In fact, the same inequality holds for any $\alpha(0 \leq \alpha \leq 1)$, since, for fixed $\phi$ and fixed $t,\left\|f_{\varepsilon}\right\|_{L^{\infty}}$ is monotone decreasing with $\alpha$.

Let \{\} denote the operator which nullifies functions over obstacles; in other words, $\left\{f_{\varepsilon}\right\}(t, x, \omega)=0$ for $x \notin Z_{\varepsilon}$, and $\left\{f_{\varepsilon}\right\}$ agrees with $f_{\varepsilon}$ otherwise. It not difficult to see that

$$
\partial_{t}\left\{f_{\varepsilon}\right\}=\left\{\partial_{t} f_{\varepsilon}\right\} \text { and } \partial_{x}\left\{f_{\varepsilon}\right\}=\left\{\partial_{x} f_{\varepsilon}\right\}+n_{x}\left(\left.f_{\varepsilon}\right|_{\partial Z_{\varepsilon}^{+}}\right) \delta_{\partial Z_{\varepsilon}}
$$

where $n_{x}$ is the inward unit normal to $\partial Z_{\varepsilon}$ at $x, \delta_{\partial Z_{\varepsilon}}$ is the Dirac delta density concentrated on $\partial Z_{\varepsilon}$, and $\left.f_{\varepsilon}\right|_{\partial Z_{\varepsilon}^{+}}$is the "jump," or exterior limit, of $f_{\varepsilon}$ at $\partial Z_{\varepsilon}$ (see, e.g., Schwartz [15], Ch. $2, \S 3$, Ex. 1). Therefore $\left\{\partial_{t} f_{\varepsilon}\right\}+c \omega \cdot\left\{\partial_{x} f_{\varepsilon}\right\}=0$ leads to

$$
\partial_{t}\left\{f_{\varepsilon}\right\}+c \omega \cdot \partial_{x}\left\{f_{\varepsilon}\right\}=c \omega \cdot n_{x}\left(\left.f_{\varepsilon}\right|_{\partial Z_{\varepsilon}^{+}}\right) \delta_{\partial Z_{\varepsilon}}
$$

Now integrating the right hand side over $[0, T] \times K \times \mathbf{S}^{1}$, we find

$$
\begin{gathered}
\int_{0}^{T} \int_{K} \int_{\mathbf{S}^{1}} c \omega \cdot n_{x}\left(f_{\varepsilon} \mid \partial Z_{\varepsilon}^{+}\right) d \omega \delta_{\partial Z_{\varepsilon}} d t \leq 2 \pi c\|\phi\|_{L^{\infty}} T \int_{K} \delta_{\partial Z_{\varepsilon}} \leq \\
2 \pi c\|\phi\|_{L^{\infty}} T\left(\frac{\operatorname{diam} K}{a \varepsilon}\right)^{n}\left|\mathbf{S}^{n-1}\right|\left(r \varepsilon^{\gamma}\right)^{n-1}=O\left(\varepsilon^{\gamma(n-1)-n}\right)
\end{gathered}
$$

from which it follows that, for every $\gamma>\frac{n}{n-1}$, we have $\partial_{t}\left\{f_{\varepsilon}\right\}+c \omega \cdot \partial_{x}\left\{f_{\varepsilon}\right\} \rightarrow 0$ in the sense of distributions as $\varepsilon \rightarrow 0$. In other words, $\gamma>\frac{n}{n-1}$ leads to a free transport equation in the macroscopic limit, so that $\gamma_{c} \leq \frac{n}{n-1}$.

\section{Ergodization Rates for Linear Flow on the Torus.}

This section is a self-contained discussion of the ergodization rates for linear flow on the torus developed in Dumas [8] and used in the proofs of the principal results announced above. We also derive an ergodization rate in the two-dimensional case which is mentioned in Remark 3.2 of [8], but is not explicitly calculated there.

\subsection{Linear Flow on $\mathbf{T}^{n}$ : Definitions and Notation.}

Given a direction vector $\omega \in \mathbf{S}^{n-1}$, we define the linear flow on $\mathbf{T}^{n} \equiv \mathbf{R}^{n} / \mathbf{Z}^{n}$ associated to $\omega$ as the family of maps

$$
\omega_{t}: \mathbf{T}^{n} \rightarrow \mathbf{T}^{n} \quad \text { given by } \quad \theta \mapsto \theta+t \omega, \quad t \in \mathbf{R} .
$$

The maps $\omega_{t}$ are well defined: if $y-y^{\prime} \in \mathbf{Z}^{n}$, then clearly $(y+t \omega)-\left(y^{\prime}+t \omega\right) \in \mathbf{Z}^{n}$; moreover, since the translations by the vector $t \omega$ form a one-parameter group of $C^{\infty}$ transformations 
of $\mathbf{R}^{n}$, by passing to the quotient, we deduce that $\omega_{t}$ defines a one-parameter group of $C^{\infty}$ transformations of $\mathbf{T}^{n}$.

A rectilinear orbit segment of $\mathbf{T}^{n}$ starting at $\theta$ is a parametrized curve of the form

$$
\bigcup_{a<t<b} \omega_{t}(\theta)
$$

where $a$ and $b$ are real numbers. Of course, the complete orbit of $\theta$ is obtained when $a=-\infty$ and $b=+\infty$.

Given $\theta \in \mathbf{T}^{n}$, there is an open neighborhood $U$ of $\theta$ in $\mathbf{T}^{n}$ diffeomorphic to an open set $V$ in $\mathbf{R}^{n}$; we equip $U$ with the pull-back metric of the Euclidean metric on $V$. In fact, it is the unique metric on $\mathbf{T}^{n}$ which is invariant under all transformations $\omega_{t}$ for all $t \geq 0$ and every $\omega \in \mathbf{S}^{n-1}$. $\mathbf{T}^{n}$ equipped with this metric is a complete Riemannian manifold called the flat torus of dimension $n$. Its geodesic curves are the rectilinear orbit segments defined above. The associated geodesic distance is

$$
\operatorname{dist}\left\{\theta, \theta^{\prime}\right\}=\inf \left\{\left|x-x^{\prime}\right| \mid \theta=x \bmod 1, \theta^{\prime}=x^{\prime} \bmod 1\right\}
$$

Fix $1 \geq R>0$ and let $\mathcal{B}_{R / 2}(\theta)$ be the ball of diameter $R$ centered on $\theta \in \mathbf{T}^{n}$. We say that the flow $\omega_{t}$ with direction vector $\omega \in \mathbf{S}^{n-1}$ ergodizes $\mathbf{T}^{n}$ to within $R$ after time $T$ if and only if

$$
\bigcup_{0 \leq t \leq T} \omega_{t}\left(\mathcal{B}_{R / 2}(\theta)\right)=\mathbf{T}^{n}
$$

for every $\theta \in T^{n}$. This condition is clearly independent of $\theta$ : for all $\theta, \phi \in \mathbf{T}^{n}$,

$$
\bigcup_{0 \leq t \leq T} \omega_{t}\left(\mathcal{B}_{R}(\theta)\right)=\bigcup_{0 \leq t \leq T} \omega_{t}\left(\mathcal{B}_{R}(\phi)\right)+\{\theta-\phi\}
$$

so that

$$
\text { if } \bigcup_{0 \leq t \leq T} \omega_{t}\left(\mathcal{B}_{R}(\phi)\right)=\mathbf{T}^{n}, \text { then } \bigcup_{0 \leq t \leq T} \omega_{t}\left(\mathcal{B}_{R}(\theta)\right)=\mathbf{T}^{n}
$$

\subsection{The Special Case of Linear Flow on $\mathbf{T}^{2}$.}

On $\mathbf{T}^{2}$, we restrict ourselves to the class of flows with directions $\omega=\left(\omega_{1}, \omega_{2}\right)$ "between $0^{\circ}$ and $45^{\circ}$; i.e., directions belonging to the eighth-circle $\mathcal{F}$ defined as

$$
\mathcal{F}=\left\{\left(\omega_{1}, \omega_{2}\right) \in \mathbf{S}^{1} \mid \sqrt{2} / 2<\omega_{1}<1 \text { and } 0<\omega_{2} / \omega_{1}<1\right\}
$$

(Note: We use $\omega_{1}, \omega_{2}$ to denote the components of the direction $\omega$, even though the subscript $t$ appears in our use of $\omega_{t}$ to designate the flow with direction $\omega$. Since we never consider the time- 1 or time- 2 maps of $\omega_{t}$, this should not cause confusion.)

Since we are only interested in directions with irrational slope (directions with rational slope do not generate ergodic flows), we have eliminated the directions $\omega=(1,0)$ and 
$\omega=(\sqrt{2} / 2, \sqrt{2} / 2)$. The entirety of linear flows on $\mathbf{T}^{2}$ is then obtained by considering the seven remaining open sectors of directions in $\mathbf{S}^{1}$ which we reduce to $\mathcal{F}$ by symmetry.

\subsection{The Correspondence Between Rotations of $\mathbf{T}^{1}=\mathbf{R} / \mathbf{Z}$ and Linear Flows on $\mathbf{T}^{2}$.}

For $0<\beta<1$, we define the rotation $\mathcal{R}_{\beta}: \mathbf{T}^{1} \rightarrow \mathbf{T}^{1}$ by $\mathcal{R}_{\beta}(x)=x+\beta \bmod 1$. We say that $\mathcal{R}_{\beta}$ fills $\mathbf{T}^{1}$ to within $R(0<R<1)$ after $N$ iterations if and only if for every $x \in \mathbf{T}^{1}$

$$
\bigcup_{k=1}^{N} \mathcal{R}_{\beta}^{k}\left(I_{R}(x)\right)=\mathbf{T}^{1}
$$

where $I_{R}(x)$ designates the closed interval of length $R$ centered at $x \in \mathbf{T}^{1}$.

There is a one-to-one correspondence between rotations on $\mathbf{T}^{1}$ and linear flows on $\mathbf{T}^{2}$ :

- For $\omega=\left(\omega_{1}, \omega_{2}\right) \in \mathcal{F}$, the Poincaré map of $\omega_{t}$ induced on a vertical section of $\mathbf{T}^{2}$ is the rotation $\mathcal{R}_{\omega_{2} / \omega_{1}}$.

- Conversely, given a rotation $\mathcal{R}_{\beta}: \mathbf{T}^{1} \rightarrow \mathbf{T}^{1}$ with $0<\beta<1$, the linear suspension of $\mathcal{R}_{\beta}$ on $\mathbf{T}^{2}$ is the linear flow $\omega_{t}: \mathbf{T}^{2} \rightarrow \mathbf{T}^{2}$ with direction $\omega=\left(\frac{1}{\sqrt{1+\beta^{2}}}, \frac{\beta}{\sqrt{1+\beta^{2}}}\right)$.

This correspondence also establishes a link between the ergodization time for linear flows of $\mathbf{T}^{2}$ and the number of iterations of the corresponding rotation necessary to fill $\mathbf{T}^{1}$ :

Lemma 1. Let $\omega=\left(\omega_{1}, \omega_{2}\right) \in \mathcal{F}$ (cf. (11)) and set $\beta=\omega_{1} / \omega_{2}$. If $\mathcal{R}_{\beta}$ fills $\mathbf{T}^{1}$ to within $R$ after $N$ iterations, then $\omega_{t}$ ergodizes $\mathbf{T}^{2}$ to within $\omega_{1} R$ after time $T=N / \omega_{1}$.

Proof. (Elementary geometry)

Remark 4. In particular, Lemma 1 shows that $\omega_{t}$ ergodizes $\mathbf{T}^{2}$ to within $R$ after time $T=\sqrt{2} N$, since $\sqrt{2} / 2<\omega_{1}<1$.

\subsection{The Ergodization Time for Flows in Two Dimensions}

We now introduce the set $\mathcal{D}_{n}(s, C)$ of "highly irrational" direction vectors satisfying Diophantine conditions:

$$
\mathcal{D}_{n}(s, C)=\left\{\left.\omega \in \mathbf{S}^{n-1}|| \omega \cdot k|\geq C| k\right|^{-s} \forall k \in \mathbf{Z}^{n} \backslash\{0\}\right\}
$$

We recall that, for $s>n-1, \mathcal{D}_{n}(s, C)$ is nonempty for small enough $C>0$, and in fact meas $\left\{\mathcal{D}_{n}(s, C)^{c}\right\} \rightarrow 0$ as $C \rightarrow 0$ (here the superscript $c$ denotes the complement in $\mathbf{S}^{n-1}$ ).

The main results here are the following theorem and its corollary.

Theorem 3. Let $0<R<1, \omega \in \mathcal{F}\left(\right.$ cf. (9)) and set $\beta=\omega_{2} / \omega_{1}$. If $\omega \in \mathcal{D}_{2}(s, C)$, then $\mathcal{R}_{\beta}$ fills $\mathbf{T}^{1}$ to within $R$ after $\left[(3 \sqrt{2})^{s} /\left(C R^{s}\right)\right]$ iterations. (Here $[x]$ is the integer part of $\left.x.\right)$

The result we use to prove Theorems $1 \mathrm{~A}$ and $1 \mathrm{~B}$ is the following

Corollary 1. $\omega \in \mathcal{D}_{2}(s, C) \Longrightarrow \omega_{t}$ ergodizes $\mathbf{T}^{2}$ to within $R$ after time $T=\frac{3^{s}(\sqrt{2})^{s+1}}{C R^{s}}$. Proof. Apply Lemma 1 to Theorem 3. 


\subsection{The Ergodization Time for Flows in Higher Dimensions}

Before proceeding to the proof of Theorem 3, for comparison we next state the best ergodization time known to us in higher dimensions $n>2$, then follow with a simple conjecture and a remark on how the class $\mathcal{D}_{n}(s, C)$ may be enlarged while maintaining the ergodization time.

Theorem 4. $\omega \in \mathcal{D}_{n}(s, C) \Longrightarrow \omega_{t}$ ergodizes $\mathbf{T}^{n}$ to within $R$ after time $T=\frac{\kappa}{C R^{s+n / 2}}$.

The constant $\kappa$ appearing in Theorem 4 depends only on $n$ and $s$, and involves the Sobolev norm of a certain "smoothest test function." It is given explicitly in [8], where complete proofs of Theorem 4 (called Theorem 1 in [8]) and related results appear.

Remark 5. Based on the way this estimate enters into the proofs of Theorems $2 \mathrm{~A}$ and $2 \mathrm{~B}$, we note that it would be consistent with $\gamma_{c}=\frac{n}{n-1}$ if the optimal ergodization time appearing above in Theorem 4 were of the form $T=\kappa /\left(C R^{s}\right)$. In fact Remark 3, above, together with the proof of Theorems $2 \mathrm{~A}$ and $2 \mathrm{~B}$ show that this is the best possible ergodization time.

Remark 6. Although we do not make use of it in this article, it is worth noting that the class of direction vectors $\omega \in \mathbf{S}^{n-1}$ whose ergodization times are comparable to those of Theorems 3 and 4 is in fact larger than $\mathcal{D}_{n}(s, C)$. This is because given a fixed $R>0$, even $\omega \in \mathbf{S}^{n-1}$ with rationally related components will fill $\mathbf{T}^{n}$ to within $R$, provided the rational relations occur at sufficiently high order. This can be quantified by defining the set of "nearly highly irrational" direction vectors satisfying Diophantine conditions truncated at order $N$ :

$$
\mathcal{D}_{n}(s, C, N)=\left\{\left.\omega \in \mathbf{S}^{n-1}|| \omega \cdot k|\geq C| k\right|^{-s} \forall k \in \mathbf{Z}^{n} \text { with } 0<|k| \leq N\right\} .
$$

It is easy to see that $\mathcal{D}_{n}(s, C)$ is a Cantor set in $\mathbf{S}^{n-1}$, and that for each $N<\infty, \mathcal{D}_{n}(s, C, N)$ is an approximating superset of $\mathcal{D}_{n}(s, C)$ consisting of finitely many connected components with nonempty interior. In higher dimensions $n>2$, it is possible to show that there exists a "critical cutoff" $N_{\text {crit }}$ such that $N \geq N_{\text {crit }}$ ensures that flows with direction vectors $\omega \in \mathcal{D}_{n}(s, C, N)$ retain the ergodization time of Theorem 4 up to a factor depending on $N$. Details and an estimate of $N_{\text {crit }}$ may be found in [8]. In two dimensions, the situation is considerably simpler: the proof of Theorem 3 which we give below shows that $N_{\text {crit }} \leq 3 / R$.

\subsection{Proof of Theorem 3.}

Proof.

Part 1. A bound on the growth of the denominators in the continued fraction expansion of $\beta=\omega_{2} / \omega_{1}$.

Let the rational number $p_{n} / q_{n}$ be the $n^{t h}$ convergent in the continued fraction expansion of $\beta=\omega_{2} / \omega_{1}$. We use the following properties of the sequence of convergents $\left\{p_{n} / q_{n}\right\}_{n=1}^{\infty}$ of $\beta \notin \mathbf{Q}, 0<\beta<1$ (cf. for example Arnold [2] or Schmidt [14]).

(i) (rational approximation) $\forall k, \quad\left|\beta-\frac{p_{k}}{q_{k}}\right|<\frac{1}{q_{k} q_{k+1}}<\frac{1}{q_{k}^{2}}$

(ii) (growth of denominators for $\beta \notin \mathbf{Q}$ ) $q_{1}=1, q_{k}<q_{k+1}$, and $q_{k} \rightarrow \infty$ 
(iii) (convergents not greater than 1) $\forall k, 1 \leq p_{k} \leq q_{k}$

According to (ii), we may choose an index $k$ so that $q_{k} \leq 3 / R<q_{k+1}$. Fixing this value of $k$, and multiplying the first inequality of (i) by $\omega_{1} q_{k}$, we obtain

$$
\begin{gathered}
\frac{1}{q_{k+1}}>\frac{\omega_{1}}{q_{k+1}}>\left|\omega_{2} q_{k}-\omega_{1} p_{k}\right|=\left|\omega \cdot\left(-p_{k}, q_{k}\right)\right| \geq C\left\|\left(-p_{k}, q_{k}\right)\right\|^{-s} \\
=C\left(p_{k}^{2}+q_{k}^{2}\right)^{-s / 2} \geq C\left(2 q_{k}^{2}\right)^{-s / 2} \geq \frac{C R^{s}}{(3 \sqrt{2})^{s}}
\end{gathered}
$$

From this we deduce that $q_{k+1}<\left[\frac{(3 \sqrt{2})^{s}}{C R^{s}}\right]$.

Part 2. Shadowing of the (periodic) orbits of $\mathcal{R}_{p_{k+1} / q_{k+1}}$ by orbits of $\mathcal{R}_{\beta}$.

Set $r=p_{k+1} / q_{k+1}$, which is rational in lowest terms. The sequence $\left\{\mathcal{R}_{r}^{j}(x)\right\} \subset \mathbf{T}^{1}$ is clearly periodic with period $q_{k+1}$ for all $x \in \mathbf{T}^{1}$. In fact, $\mathcal{R}_{r}$ fills $\mathbf{T}^{1}$ to within exactly $1 / q_{k+1}<R / 3$ after $q_{k+1}$ iterations. It is easy to see that the first $q_{k+1}$ iterations of $\mathcal{R}_{r}$ are shadowed by those of $\mathcal{R}_{\beta}$.

More precisely: for every $1 \leq j \leq q_{k+1}$ and all $x \in \mathbf{T}^{1}$, we have:

$$
\operatorname{dist}\left\{\mathcal{R}_{r}^{j}(x), \mathcal{R}_{\beta}^{j}(x)\right\} \leq\left|j\left(\frac{p_{k+1}}{q_{k+1}}-\frac{\omega_{2}}{\omega_{1}}\right)\right|<q_{k+1} \frac{1}{q_{k+1}^{2}}=\frac{1}{q_{k+1}}<R / 3
$$

Now, since $\mathcal{R}_{r}$ fills $\mathbf{T}^{1}$ to within $R / 3$ after $q_{k+1}$ iterations, and since each iterate $\mathcal{R}_{r}^{j}(x)$ is shadowed to within $R / 3$ by $\mathcal{R}_{\beta}^{j}(x)\left(1 \leq j \leq q_{k+1}\right)$, it follows that $\mathcal{R}_{\beta}$ fills $\mathbf{T}^{1}$ to within $R$ after $q_{k+1}<\left[\frac{(3 \sqrt{2})^{s}}{C R^{s}}\right]$ iterations. $/ /$

Remark 7. In Part 1 above, whether $\beta=\omega_{2} / \omega_{1}$ is rational (so that its continued fraction expansion terminates) or irrational, it is still possible to choose $k$ so that $q_{k} \leq 3 / R<q_{k+1}$ provided $\omega \in \mathcal{D}_{n}(s, C, N)$ with $N \geq 3 / R$, as the reader may easily check. This establishes the last part of Remark 6 , above (since the rest of the proof goes through unchanged).

\section{Proofs of Principal Results.}

The proofs of Theorems 1A,B and 2A,B above differ only in the estimate of the ergodization time, which in turn depends on the dimension of the ambient space. We therefore give complete details of the proofs in the two-dimensional cases only (Theorems $1 \mathrm{~A}$ and $1 \mathrm{~B})$.

6.1 Proof of Theorem $1 \mathrm{~A}$.

Proof. We begin by estimating the measure of the complement of $\mathcal{D}_{2}(s, C)$ in $\mathbf{S}^{1}$ (with respect to the uniform measure $\mu$ on $\mathbf{S}^{1}$ ). From elementary geometry, we have

$$
\mu\left\{\mathcal{D}_{2}(s, C)^{c}\right\}=\mu\left\{\mathbf{S}^{1} \backslash \mathcal{D}_{2}(s, C)\right\} \leq K_{1} \sum_{0 \neq k \in \mathbf{Z}^{2}} \frac{C}{|k|^{s+1}}=C K_{1} \rho(s+1)
$$


for some constant $K_{1}>0$, and where $\rho(s+1)=\sum_{0 \neq k \in \mathbf{Z}^{2}}|k|^{-(s+1)}$ converges for $s>1$.

It is clear (see the definition) that if the flow $\omega_{t}$ with direction vector $\omega$ ergodizes $\mathbf{T}_{a \varepsilon}^{2} \equiv \mathbf{R}^{2} /(a \varepsilon \mathbf{Z})^{2}$ to within $2 r \varepsilon^{\gamma}$ after time $T_{\varepsilon}$, then $\forall x \in Z_{\varepsilon}$ the collision time $\tau_{\varepsilon}(x, \omega) \leq$ $T_{\varepsilon}$. We showed in the previous section (see Corollary 1) that every flow with direction $\omega \in \mathcal{D}_{2}(s, C)$ ergodizes $\mathbf{T}^{2}=\mathbf{R}^{2} / \mathbf{Z}^{2}$ to within $\frac{2 r}{a} \varepsilon^{\gamma-1}$ after time $3^{s}(\sqrt{2})^{s+1} C^{-1}\left(\frac{2 r}{a} \varepsilon^{\gamma-1}\right)^{-s}$. Consequently, every vector $\omega \in \mathcal{D}_{2}(s, C)$ ergodizes $\mathbf{T}_{a \varepsilon}^{2}=\mathbf{R}^{2} /(a \varepsilon \mathbf{Z})^{2}$ to within $2 r \varepsilon^{\gamma}$ after time

$$
T_{\varepsilon}=a \varepsilon \frac{3^{s}(\sqrt{2})^{s+1}}{C\left(\frac{2 r}{a} \varepsilon^{\gamma-1}\right)^{s}}=a^{1+(\gamma-1) s} \frac{3^{s}(\sqrt{2})^{s+1}}{C(2 r)^{s}} \varepsilon^{1-(\gamma-1) s} .
$$

Now let $s$ be such that $1<s<(\gamma-1)^{-1}$. According to the formula above, at any $x \in Z_{\varepsilon}$, directions $\omega$ belonging to $\mathcal{D}_{2}(s, C)$ have collision times $\tau_{\varepsilon}(x, \omega)$ which we estimate as

$$
(x, \omega) \in Z_{\varepsilon} \times \mathcal{D}_{2}(s, C) \Longrightarrow \tau_{\varepsilon}(x, \omega) \leq \frac{K_{2}}{C} \varepsilon^{1-(\gamma-1) s},
$$

where

$$
K_{2}=a^{1+(\gamma-1) s} \frac{3^{s}(\sqrt{2})^{s+1}}{c(2 r)^{s}}
$$

and we see that $\tau_{\varepsilon}$ vanishes together with $\varepsilon$ on $Z_{\varepsilon} \times \mathcal{D}_{2}(s, C)$. We therefore decompose the initial data $\phi$ into the disjoint sum

$$
\phi=\phi \mathbf{1}_{\mathcal{D}_{2}(s, C)}+\phi \mathbf{1}_{\mathcal{D}_{2}(s, C)^{c}}
$$

and we consider the corresponding decomposition of the solution of (5)-(6)-(7A)

$$
f_{\varepsilon}=g_{\varepsilon} \mathbf{1}_{\mathcal{D}_{2}(s, C)}+h_{\varepsilon} \mathbf{1}_{\mathcal{D}_{2}(s, C)^{c}}
$$

It follows that

$$
\begin{gathered}
\int_{0}^{T} \int_{K \cap Z_{\varepsilon}} \int_{\mathbf{S}^{1}}\left|f_{\varepsilon}(t, x, \omega)\right| d \omega d x d t= \\
\int_{0}^{T} \int_{K \cap Z_{\varepsilon}} \int_{\mathcal{D}_{2}(s, C)}\left|g_{\varepsilon}(t, x, \omega)\right| d \omega d x d t+\int_{0}^{T} \int_{K \cap Z_{\varepsilon}} \int_{\mathcal{D}_{2}(s, C)^{c}}\left|h_{\varepsilon}(t, x, \omega)\right| d \omega d x d t \leq \\
\frac{K_{2}}{C} \varepsilon^{1-(\gamma-1) s} \operatorname{meas}\{K\} 2 \pi\|\phi\|_{L^{\infty}}+T \operatorname{meas}\{K\} C K_{1} \rho(1+s)\|\phi\|_{L^{\infty}} \leq \\
\frac{A}{2}\left(C+\frac{\varepsilon^{1-(\gamma-1) s}}{C}\right)\|\phi\|_{L^{\infty}}
\end{gathered}
$$

where

$$
A=2 \operatorname{meas}\{K\} \cdot \max \left\{T K_{1} \rho(1+s), 2 \pi K_{2}\right\} .
$$

The bound on the right-hand side of (31) is minimized by choosing $C=\varepsilon^{(1+s-\gamma s) / 2}$ (the so-called "distinguished limit"), in which case the integral on the left-hand side of (31) is bounded by $A \varepsilon^{(1+s-\gamma s) / 2}\|\phi\|_{L^{\infty}}$, as desired. // 
Remark 8. If, instead of Theorems $1 \mathrm{~A}$ and $2 \mathrm{~A}$ as stated (with their order estimates of the rate of decay of the integral with $\varepsilon$ ), one wishes to prove only that

$$
\lim _{\varepsilon \rightarrow 0} \int_{0}^{T} \int_{K \cap Z_{\varepsilon}} \int_{\mathbf{S}^{n-1}}\left|f_{\varepsilon}(t, x, \omega)\right| d \omega d x d t=0
$$

it suffices to note (see the first part of the proof above for $n=2$ ) that

$$
\operatorname{meas} \bigcap_{\substack{s>n-1 \\ C>0}}\left(\mathcal{D}_{n}(s, C)\right)^{c}=0
$$

from which it follows that for almost every $(x, \omega) \in Z_{\varepsilon} \times \mathbf{S}^{n-1}$, the time to collision $\tau_{\varepsilon}(x, \omega)$ vanishes with $\varepsilon$.

\subsection{Proof of Theorem $1 B$.}

Because it is somewhat longer than the proof just given, we break the proof of Theorem 1B into Parts a) through $f$ ).

\section{Proof.}

a) Solution of the Transport Equation

We denote by $\left(X_{\varepsilon}(t, x, \omega), \Omega_{\varepsilon}(t, x, \omega)\right)$ the "reverse broken flow" of the system $(5)-(6)$ (7B) on $\overline{Z_{\varepsilon}} \times \mathbf{S}^{1}$. More explicitly, using the method of characteristics we may write this flow as the solution of the system

$$
\begin{gathered}
\frac{d X_{\varepsilon}}{d t}=-\Omega_{\varepsilon}, \quad \frac{d \Omega_{\varepsilon}}{d t}=0, \quad X_{\varepsilon} \in Z_{\varepsilon} \\
X_{\varepsilon}(0, x, \omega)=x, \quad \Omega_{\varepsilon}(0, x, \omega)=\omega \\
X_{\varepsilon}(t+0)=X_{\varepsilon}(t-0), \quad \Omega_{\varepsilon}(t+0)=\mathcal{R}\left(n_{X_{\varepsilon}}\right) \Omega_{\varepsilon}(t-0), \quad X_{\varepsilon} \in \partial Z_{\varepsilon} .
\end{gathered}
$$

We denote by $\mathcal{N}_{\varepsilon}(t, x, \omega)=\operatorname{card}\left\{0 \leq \tau<t \mid X_{\varepsilon}(\tau, x, \omega) \in \partial Z_{\varepsilon}\right\}$ the number of collisions experienced by a particle with initial condition $(x, \omega)$, moving backward in $\bar{Z}_{\varepsilon}$ for a time $t>0$. The value of $f_{\varepsilon}$ after time $t$ is then

$$
f_{\varepsilon}(t, x, \omega)=\alpha^{\mathcal{N}_{\varepsilon}(t, x, \omega)} \phi\left(X_{\varepsilon}(t, x, \omega), \Omega_{\varepsilon}(t, x, \omega)\right)
$$

b) Decomposition of the Solution

As in the proof of Theorem $1 \mathrm{~A}$, we decompose the solution $f_{\varepsilon}$ into a disjoint sum of Diophantine and non-Diophantine directions:

$$
f_{\varepsilon}=g_{\varepsilon}+h_{\varepsilon}, \quad \text { where } \quad g_{\varepsilon}=f_{\varepsilon} \mathbf{1}_{\mathcal{D}_{2}\left(s, C_{0}\right)}, \quad h_{\varepsilon}=f_{\varepsilon} \mathbf{1}_{\mathcal{D}_{2}\left(s, C_{0}\right)^{c}},
$$

and where $C_{0}>0$ will be determined later. 
c) Decomposition of the Integral

As in the proof of Theorem $1 \mathrm{~A}$, we write

$$
\begin{aligned}
& \left|\int_{0}^{T} \int_{K \cap Z_{\varepsilon}} \int_{\mathbf{S}^{1}}\right| f_{\varepsilon}(t, x, \omega)|d \omega d x d t| \leq \\
& T\|\phi\|_{L^{\infty}} \operatorname{meas}\{K\} C_{0} K_{1} \rho(1+s)+\int_{0}^{T} \int_{K \cap Z_{\varepsilon}} \int_{\mathcal{D}_{2}\left(s, C_{0}\right)}\left|g_{\varepsilon}(t, x, \omega)\right| d \omega d x d t \leq \\
& T\|\phi\|_{L^{\infty}} \operatorname{meas}\{K\} C_{0} K_{1} \rho(1+s)+\|\phi\|_{L^{\infty}} \int_{0}^{T} \int_{K \cap Z_{\varepsilon}} \int_{\mathcal{D}_{2}\left(s, C_{0}\right)} \alpha^{\mathcal{N}_{\varepsilon}(t, x, \omega)} d \omega d x d t .
\end{aligned}
$$

Now we know that $(x, \omega) \in Z_{\varepsilon} \times \mathcal{D}_{2}\left(s, C_{0}\right) \Longrightarrow \tau_{\varepsilon}(x, \omega) \leq K_{2} C_{0}^{-1} \varepsilon^{1-(\gamma-1) s} \equiv T_{\varepsilon}^{0}$ (cf. Eqns. (26)-(28)). We shall later choose $C_{0}$ so that $T_{\varepsilon}^{0} \rightarrow 0$ as $\varepsilon \rightarrow 0$, so we may assume that $\varepsilon_{0}$ is small enough to ensure that $2 T_{\varepsilon}^{0} \leq T$ for $0<\varepsilon \leq \varepsilon_{0}$. We then split the last integral into an integration over the time intervals $\left[0,2 T_{\varepsilon}^{0}\right]$ and $\left[2 T_{\varepsilon}^{0}, T\right]$ as follows:

$$
\begin{gathered}
\int_{0}^{T} \int_{K \cap Z_{\varepsilon}} \int_{\mathcal{D}_{2}\left(s, C_{0}\right)} \alpha^{\mathcal{N}_{\varepsilon}(t, x, \omega)} d \omega d x d t= \\
\int_{0}^{2 T_{\varepsilon}^{0}} \int_{K \cap Z_{\varepsilon}} \int_{\mathcal{D}_{2}\left(s, C_{0}\right)} \alpha^{\mathcal{N}_{\varepsilon}(t, x, \omega)} d \omega d x d t+\int_{2 T_{\varepsilon}^{0}}^{T} \int_{K \cap Z_{\varepsilon}} \int_{\mathcal{D}_{2}\left(s, C_{0}\right)} \alpha^{\mathcal{N}_{\varepsilon}(t, x, \omega)} d \omega d x d t .
\end{gathered}
$$

The first integral on the right hand side of (41) is easily bounded by $2 T_{\varepsilon}^{0}$ meas $\{K\} 2 \pi=$ $2 K_{2} C_{0}^{-1} \varepsilon^{1-(\gamma-1) s}$ meas $\{K\} 2 \pi$. As for the second integral on the right hand side of (41), we modify the argument of $\mathcal{N}_{\varepsilon}$ in two ways which do not increase $\mathcal{N}_{\varepsilon}$.

First, for all $(x, \omega) \in\left(K \cap Z_{\varepsilon}\right) \times \mathcal{D}_{2}\left(s, C_{0}\right)$, we have $\tau_{\varepsilon}(x, \omega) \leq T_{\varepsilon}^{0}$; in particular, for $\operatorname{such}(x, \omega)$ and for all $t \in\left[2 T_{\varepsilon}^{0}, T\right]$, we have $t>\tau_{\varepsilon}(x, \omega)$, so that on the interval of integration $\left[2 T_{\varepsilon}^{0}, T\right]$, we may shift the time by $\tau_{\varepsilon}(x, \omega)$ and particles along their trajectories a distance $c \tau_{\varepsilon}(x, \omega)$ to obtain

$$
\mathcal{N}_{\varepsilon}(t, x, \omega) \geq \mathcal{N}_{\varepsilon}\left(t-\tau_{\varepsilon}(x, \omega), x-c \tau_{\varepsilon}(x, \omega) \omega, \omega\right)
$$

Second, for all $t \in\left[2 T_{\varepsilon}^{0}, T\right]$ and all $(x, \omega) \in\left(K \cap Z_{\varepsilon}\right) \times \mathcal{D}_{2}\left(s, C_{0}\right)$, we have $t / 2 \geq \tau_{\varepsilon}(x, \omega)$. Since $\mathcal{N}_{\varepsilon}$ is monotone increasing in its first argument, it follows that for such $(t, x, \omega)$,

$$
\mathcal{N}_{\varepsilon}\left(t-\tau_{\varepsilon}(x, \omega), x-c \tau_{\varepsilon}(x, \omega) \omega, \omega\right) \geq \mathcal{N}_{\varepsilon}\left(t / 2, x-c \tau_{\varepsilon}(x, \omega) \omega, \omega\right)
$$

The right hand side of Eq. (41) is therefore bounded by

$$
2 T_{\varepsilon}^{0} \operatorname{meas}\{K\} 2 \pi+I_{\varepsilon}=2 \frac{K_{2}}{C_{0}} \varepsilon^{1-(\gamma-1) s} \operatorname{meas}\{K\} 2 \pi+I_{\varepsilon},
$$

where

$$
I_{\varepsilon}=\int_{2 T_{\varepsilon}^{\mathrm{o}}}^{T} \int_{K \cap Z_{\varepsilon}} \int_{\mathcal{D}_{2}\left(s, C_{0}\right)} \alpha^{\mathcal{N}_{\varepsilon}\left(t / 2, x-c \tau_{\varepsilon}(x, \omega) \omega, \omega\right)} d \omega d x d t
$$




\section{d) Passage Through Reflection}

We introduce the periodicized (or punctured toroidal) domain $Y_{\varepsilon}$ associated to $Z_{\varepsilon}$ by writing $Y_{\varepsilon}=Z_{\varepsilon} /(a \varepsilon \mathbf{Z})^{2}$. We note that $Y_{\varepsilon}$ has compact closure, and that its boundary is a single circular obstacle of radius $r \varepsilon^{\gamma}$. We also define the boundary domains $\Delta_{\varepsilon}^{+}$and $\Delta_{\varepsilon}^{+}(s, C)$ by

$$
\begin{gathered}
\Delta_{\varepsilon}^{+}=\left\{(y, \omega) \in \partial Y_{\varepsilon} \times \mathbf{S}^{1} \mid \omega \cdot n_{y}>0\right\}, \\
\Delta_{\varepsilon}^{+}(s, C)=\Delta_{\varepsilon}^{+} \cap\left(\partial Y_{\varepsilon} \times \mathcal{D}_{2}\left(s, C_{0}\right)\right),
\end{gathered}
$$

where $n_{y}$ is the inward normal at the point $y \in \partial Y_{\varepsilon}$.

Now let

$$
\mathcal{Q}_{\varepsilon}=\left\{(y, \omega) \in \partial Y_{\varepsilon} \times \mathbf{S}^{1} \mid \exists k \in \mathbf{Z}^{2} \backslash\{0\} \text { s.t. } \mathcal{R}\left(n_{y}\right) \omega \cdot k=0\right\} .
$$

The set $\mathcal{Q}_{\varepsilon}$ is clearly of $d y d \omega$-measure 0 . On the other hand, linear flows on the torus with irrational slope are topologically transitive (i.e. the orbit of each point is dense). It follows in particular that for all $(y, \omega) \notin \mathcal{Q}_{\varepsilon}$, one has $\tau_{\varepsilon}\left(y, \mathcal{R}\left(n_{y}\right) \omega\right)<+\infty$. We may then define the boundary (or reflection) map

$$
\mathcal{T}: \Delta_{\varepsilon}^{+} \backslash \mathcal{Q}_{\varepsilon} \rightarrow \Delta_{\varepsilon}^{+} \quad \text { by } \quad \mathcal{T}(y, \omega)=\left(y-\tau_{\varepsilon}\left(y, \mathcal{R}\left(n_{y}\right) \omega\right) \mathcal{R}\left(n_{y}\right) \omega, \mathcal{R}\left(n_{y}\right) \omega\right)
$$

and the "nice" set

$$
\mathcal{E}_{\varepsilon}^{N}(s, C)=\bigcap_{k=0}^{N} \mathcal{T}^{-k}\left(\Delta_{\varepsilon}^{+}(s, C)\right)
$$

consisting of elements $(y, \omega) \in \Delta_{\varepsilon}^{+}(s, C) \backslash \mathcal{Q}_{\varepsilon}$ whose first $N$ iterates under $\mathcal{T}$ belong to $\Delta_{\varepsilon}^{+}(s, C)$.

Now consider the measure $d \nu_{\varepsilon}=\left|\omega \cdot n_{y}\right| d y d \omega$ on $\Delta_{\varepsilon}^{+}$. We have

$$
\begin{gathered}
\nu_{\varepsilon}\left(\mathcal{E}_{\varepsilon}^{N}(s, C)^{c}\right)=\nu_{\varepsilon}\left(\Delta_{\varepsilon}^{+} \backslash \mathcal{E}_{\varepsilon}^{N}(s, C)\right)=\nu_{\varepsilon}\left(\bigcup_{k=0}^{N} \mathcal{T}^{-k}\left(\Delta_{\varepsilon}^{+}(s, C)^{c}\right)\right) \leq \\
\sum_{k=0}^{N} \nu_{\varepsilon}\left(\mathcal{T}^{-k}\left(\Delta_{\varepsilon}^{+}(s, C)^{c}\right)\right)=(N+1) \nu_{\varepsilon}\left(\Delta_{\varepsilon}^{+}(s, C)^{c}\right) \leq(N+1) 2 \pi r \varepsilon^{\gamma} C K_{1} \rho(s+1),
\end{gathered}
$$

where we have used the fact that $\mathcal{T}$ preserves the measure $\nu_{\varepsilon}$ (see, e.g., Bunimovich-Sinai [4]).

e) Further Estimates

We return to the problem of estimating the integral $I_{\varepsilon}$ in Eq. (45). First, we define the "lift" $\mathcal{A}_{\varepsilon}^{N}(s, C) \subset Z_{\varepsilon} \times \mathbf{S}^{1}$ of the nice set $\mathcal{E}_{\varepsilon}^{N}(s, C)$ by

$$
\mathcal{A}_{\varepsilon}^{N}(s, C)=\left\{(x, \omega) \in Z_{\varepsilon} \times \mathbf{S}^{1} \mid[(x, \omega)] \in \mathcal{E}_{\varepsilon}^{N}(s, C)\right\},
$$

where $[(x, \omega)]$ is the equivalence class of $(x, \omega)$ for the relation $(x, \omega) \sim\left(x^{\prime}, \omega\right) \Longleftrightarrow x-x^{\prime} \in$ $(a \varepsilon \mathbf{Z})^{2}$. 
We introduce an $\varepsilon$-dependent order parameter $C_{1}>0$ (to be chosen later so that $C_{1} \rightarrow 0$ as $\varepsilon \rightarrow 0$ ), and we separate $I_{\varepsilon}$ into an integral over initial conditions giving rise to trajectories whose directions after one collision belong to the nice set $\mathcal{A}_{\varepsilon}^{N}\left(s, C_{1}\right)$, and an integral over the complement of those initial conditions. In other words,

$$
I_{\varepsilon} \leq J^{\varepsilon}+L^{\varepsilon}
$$

where

$$
\begin{aligned}
J^{\varepsilon} & =\int_{0}^{T} \int_{K \cap Z_{\varepsilon}} \int_{\mathcal{D}_{2}\left(s, C_{0}\right)} \alpha^{\mathcal{N}_{\varepsilon}\left(t / 2, x-c \tau_{\varepsilon}(x, \omega) \omega, \omega\right)} \mathbf{1}_{\left\{\left(x-c \tau_{\varepsilon}(x, \omega) \omega, \omega\right) \in \mathcal{A}_{\varepsilon}^{N}\left(s, C_{1}\right)\right\}} d \omega d x d t, \\
L^{\varepsilon} & =\int_{0}^{T} \int_{K \cap Z_{\varepsilon}} \int_{\mathcal{D}_{2}\left(s, C_{0}\right)} \alpha^{\mathcal{N}_{\varepsilon}\left(t / 2, x-c \tau_{\varepsilon}(x, \omega) \omega, \omega\right)} \mathbf{1}_{\left\{\left(x-c \tau_{\varepsilon}(x, \omega) \omega, \omega\right) \in\left(\mathcal{A}_{\varepsilon}^{N}\left(s, C_{1}\right)\right)^{c}\right\}} d \omega d x d t .
\end{aligned}
$$

In order to estimate $J^{\varepsilon}$, we introduce yet another $\varepsilon$-dependent order parameter $\theta_{\varepsilon}$, to be chosen shortly, and we write

$$
J^{\varepsilon} \quad=J_{1}^{\varepsilon}+J_{2}^{\varepsilon},
$$

where

$$
J_{1}^{\varepsilon}=\int_{0}^{\theta_{\varepsilon}} \int_{K \cap Z_{\varepsilon}} \int_{\mathcal{D}_{2}\left(s, C_{0}\right)} \alpha^{\mathcal{N}_{\varepsilon}\left(t / 2, x-c \tau_{\varepsilon}(x, \omega) \omega, \omega\right)} \mathbf{1}_{\left\{\left(x-c \tau_{\varepsilon}(x, \omega) \omega, \omega\right) \in \mathcal{A}_{\varepsilon}^{N}\left(s, C_{1}\right)\right\}} d \omega d x d t
$$

and

$$
J_{2}^{\varepsilon}=\int_{\theta_{\varepsilon}}^{T} \int_{K \cap Z_{\varepsilon}} \int_{\mathcal{D}_{2}\left(s, C_{0}\right)} \alpha^{\mathcal{N}_{\varepsilon}\left(t / 2, x-c \tau_{\varepsilon}(x, \omega) \omega, \omega\right)} \mathbf{1}_{\left\{\left(x-c \tau_{\varepsilon}(x, \omega) \omega, \omega\right) \in \mathcal{A}_{\varepsilon}^{N}\left(s, C_{1}\right)\right\}} d \omega d x d t .
$$

We set $\theta_{\varepsilon}=2 K_{2} N C_{1}^{-1} \varepsilon^{1-(\gamma-1) s}$, where $N$ and $C_{1}$ will be chosen later so that $N \rightarrow \infty$, $C_{1} \rightarrow 0$, and $\theta_{\varepsilon} \rightarrow 0$ as $\varepsilon \rightarrow 0$. We restrict $\varepsilon_{0}>0$ so that $0<\varepsilon \leq \varepsilon_{0} \Longrightarrow \theta_{\varepsilon} \leq T$. We have

$$
J_{1}^{\varepsilon} \leq \theta_{\varepsilon} \operatorname{meas}\{K\} 2 \pi=2 K_{2} N C_{1}^{-1} \varepsilon^{1-(\gamma-1) s} \operatorname{meas}\{K\} 2 \pi .
$$

On the other hand, if $t \geq \theta_{\varepsilon}$ and if $(x, \omega) \in \mathcal{A}_{\varepsilon}^{N}\left(s, C_{1}\right)$, then because of the way $\theta_{\varepsilon}$ is defined, at least $N$ collisions occur on the interval $[0, t / 2]$; i.e., $(t, x, \omega) \in\left[\theta_{\varepsilon}, T\right] \times \mathcal{A}_{\varepsilon}^{N}\left(s, C_{1}\right) \Longrightarrow$ $\mathcal{N}_{\varepsilon}(t / 2, x, \omega) \geq N$. Therefore

$$
J_{2}^{\varepsilon} \leq T \alpha^{N} \operatorname{meas}\{K\} 2 \pi
$$

In order to estimate the integral $L^{\varepsilon}$ in Eq. (54), we are going to define a new compact set $K^{\prime}$ which is slightly bigger than $K$, and which encompasses the first collisions of all particles emanating from $K$ in the directions $\mathcal{D}_{2}\left(s, C_{0}\right)$. By restricting $\varepsilon_{0}$ such that $0<$ $\varepsilon \leq \varepsilon_{0} \Longrightarrow c T_{\varepsilon}^{0} \leq 1$, we ensure that no particle travels more than unit distance on the time interval $\left[0, T_{\varepsilon}^{0}\right]$. We may then take

$$
K^{\prime}=\left\{x \in \mathbf{R}^{2} \mid \operatorname{dist}\{x, K\} \leq 1\right\} \text {. }
$$


Let $d \lambda_{\varepsilon}$ be the image measure of $d x d \omega$ under the map $(x, \omega) \mapsto\left(x-c \tau_{\varepsilon}(x, \omega) \omega, \omega\right)$. In other words, $d \lambda_{\varepsilon}$ is the measure on $\Gamma_{\varepsilon}^{+}$given by $d \lambda_{\varepsilon}=\tau_{\varepsilon}^{+}(x, \omega)\left(n_{x} \cdot \omega\right) d x d \omega$, where $\tau_{\varepsilon}^{+}(x, \omega)=\tau_{\varepsilon}(x,-\omega)$ is the "forward" time to collision, and $n_{x}$ is the inward unit normal to $\partial Z_{\varepsilon}$ at $x$. Below we shall also denote $\Gamma_{\varepsilon}^{+}\left(s, C_{0}\right)=\Gamma_{\varepsilon}^{+} \cap\left[\left(\partial Z_{\varepsilon} \cap K^{\prime}\right) \times \mathcal{D}_{2}\left(s, C_{0}\right)\right]$. With these conventions, we see that the image of $\left(K \cap Z_{\varepsilon}\right) \times \mathcal{D}_{2}\left(s, C_{0}\right)$ under the map $(x, \omega) \mapsto\left(x-c \tau_{\varepsilon}(x, \omega) \omega, \omega\right)$ is contained in $\Gamma_{\varepsilon}^{+}\left(s, C_{0}\right)$, and we may therefore write

$$
L^{\varepsilon} \leq \int_{0}^{T} \int_{\Gamma_{\varepsilon}^{+}\left(s, C_{0}\right) \cap\left(\mathcal{A}_{\varepsilon}^{N}\left(s, C_{1}\right)\right)^{c}} \alpha^{\mathcal{N}_{\varepsilon}(t / 2, x, \omega)} d \lambda_{\varepsilon} d t .
$$

Rewriting $L^{\varepsilon}$ in this way amounts to a change of variables that is well known in the theory of neutron transport; see Cessenat [7]. Since the compact set $K^{\prime}$ contains no more than $\left(\operatorname{diam} K^{\prime} / a \varepsilon\right)^{2}$ obstacles, it follows that

$$
L^{\varepsilon} \leq T \frac{K_{2}}{C_{0}} \varepsilon^{1-(\gamma-1) s}\left(\frac{\operatorname{diam} K^{\prime}}{a \varepsilon}\right)^{2}(N+1) 2 \pi r \varepsilon^{\gamma} C_{1} K_{1} \rho(s+1) .
$$

f) Order Parameters and Final Estimate

In view of the inequalities and Eqs. (40) - (63), we have shown that the following bound holds:

$$
\begin{gathered}
\left|\int_{0}^{T} \int_{K \cap Z_{\varepsilon}} \int_{\mathbf{S}^{1}}\right| f_{\varepsilon}(t, x, \omega)|d \omega d x d t| \leq \\
\|\phi\|_{L^{\infty}}\left(K_{3} C_{0}+K_{4} C_{0}^{-1} \varepsilon^{1-(\gamma-1) s}+J_{1}^{\varepsilon}+J_{2}^{\varepsilon}+L^{\varepsilon}\right) \leq \\
\|\phi\|_{L^{\infty}}\left(K_{3} C_{0}+K_{4} \frac{\varepsilon^{1-(\gamma-1) s}}{C_{0}}+K_{4} \frac{N \varepsilon^{1-(\gamma-1) s}}{C_{1}}+K_{5} \alpha^{N}+K_{6} \frac{N C_{1}}{C_{0}} \varepsilon^{(\gamma-1)(1-s)}\right),
\end{gathered}
$$

where

$$
\begin{gathered}
K_{3}=T K_{1} \rho(s+1) \operatorname{meas}\{K\}, \quad K_{4}=4 \pi K_{2} \operatorname{meas}\{K\} \\
K_{5}=2 \pi T \operatorname{meas}\{K\}, \quad K_{6}=4 \pi r T K_{1} K_{2} \rho(s+1)\left(\operatorname{diam} K^{\prime} / a\right)^{2} .
\end{gathered}
$$

We now choose the order parameters and constants $C_{0}, C_{1}, N, b, B, s$ and $\varepsilon_{0}$ so that the integral in (64) vanishes with $\varepsilon$, and the conclusion of Theorem 1B holds. By hypothesis, $1 \leq \gamma<2$. First, choose $b>0$ such that $0<b<(2-\gamma) / 9<(2-\gamma) / 8$. For $\gamma=1$, the desired inequalities below hold for any $s>1$; for $1<\gamma<2$, set $s=$ $(2 b+\gamma-1) /(\gamma-1)>1$. Finally, take

$$
C_{0}=\varepsilon^{2-\gamma-8 b}, \quad C_{1}=\varepsilon^{2-\gamma-4 b}, \quad N=\varepsilon^{-b},
$$

and restrict $0<\varepsilon_{0}<1$ so that $0<\varepsilon \leq \varepsilon_{0} \Longrightarrow \alpha^{N}=\alpha^{\varepsilon^{-b}} \leq \varepsilon^{b}$. Then, taking all restrictions on $\varepsilon_{0}$ into account, for $0<\varepsilon \leq \varepsilon_{0}$ it is not difficult to verify that $C_{0} \rightarrow 0$, $C_{1} \rightarrow 0, N \rightarrow \infty, T_{\varepsilon}^{0} \rightarrow 0, \theta_{\varepsilon} \rightarrow 0$, as $\varepsilon \rightarrow 0$, and that

$$
\left|\int_{0}^{T} \int_{K \cap Z_{\varepsilon}} \int_{\mathbf{S}^{1}}\right| f_{\varepsilon}(t, x, \omega)|d \omega d x d t| \leq B \varepsilon^{b}\|\phi\|_{L^{\infty}}
$$


where

$$
B=K_{3}+2 K_{4}+K_{5}+K_{6} .
$$

Remark 9. In order to carry out the proofs of Theorems $2 \mathrm{~A}$ and $2 \mathrm{~B}$, it suffices to repeat the proofs just given, replacing as appropriate the estimate of the ergodization time in two dimensions (Section 5, Corollary 1) with the estimate in higher dimensions (Section 5 , Theorem 4).

Acknowledgments. We thank Claude Bardos for his encouragement, for many helpful discussions, and for pointing out to us the contents of Remarks 2 and 8. One of us (HSD) thanks the University of Paris 7 and the CMLA at the ENS-Cachan for their hospitality while much of this research was carried out.

\section{References.}

[1] R. Alexandre and K. Hamdache, Homogénéisation d'équations cinétiques en milieu perforé, C.R. Acad. Sci. 313, 339-344 (1991).

[2] V.I. Arnold, Geometrical Methods in the Theory of Ordinary Differential Equations, Springer-Verlag, New York, 1983.

[3] C. Boldighrini, L.A. Bunimovich, and Ya. G. Sinai, On the Boltzmann equation for the Lorentz gas, J. Stat. Phys. 32 (3), 477-501, (1983).

[4] L.A. Bunimovich and Ya. G. Sinai, Markov Partitions of Dispersed Billiards, Commun. Math. Phys. 73, 247-280, (1980); and Statistical properties of the Lorentz gas with periodic configurations of scatterers, Commun. Math. Phys. 78, 479-497 (1981).

[5] L.A. Bunimovich, Ya. G. Sinai, and N.I. Chernov, Markov partitions for two-dimensional hyperbolic billiards, Russian Math. Surveys 45 (3), 105-152 (1990); and Statistical properties of two-dimensional hyperbolic billiards, Russian Math. Surveys 46 (4), 47-106 (1991).

[6] J.W.S. Cassels, An Introduction to Diophantine Approximation, Cambridge (Eng.) University Press, 1957.

[7] M. Cessenat, Théorèmes de trace $L^{p}$ pour les espaces de fonctions de la neutronique, C.R. Acad. Sci. 300, 89-92 (1985).

[8] H.S. Dumas, Ergodization rates for linear flow on the torus, J. Dynamics Diff. Equations 3, 593-610 (1991).

[9] G. Galavotti, Rigorous theory of the Boltzmann equation in the Lorentz gas, Nota Interna No. 358, Istituto di Fisica, Università di Roma (1972).

[10] F. Golse, Transport dans les milieux composites fortement contrastés I: le modèle du billard, to appear in Annales Inst. Henri Poincaré. 
[11] R. Illner and M. Pulvirenti, Global validity of the Boltzmann equation for a twodimensional rare gas in a vacuum, Commun. Math. Phys. 105, 189-203, (1986).

[12] O.E. Lanford III, Time evolution of large classical systems, in Dynamical Systems, Theory and Applications (Lecture Notes in Physics no. 38, J. Moser, Ed.) 1-111, Springer-Verlag, New York, 1975.

[13] M. Pulvirenti, Global validity of the Boltzmann equation for a three-dimensional rare gas in a vacuum, Commun. Math. Phys. 113, 79-85, (1987).

[14] W.M. Schmidt, Diophantine Approximation (Lecture Notes in Mathematics No. 785), Springer-Verlag, Berlin, 1980.

[15] L. Schwartz, Théorie des distributions, Hermann, Paris, 1966.

[16] H. Spohn, The Lorentz flight process converges to a random flight process, Commun. Math. Phys. 60, 277-290 (1978). 